04

\title{
Структура магнитных флуктуаций плазмы в торсатроне Ураган-3М при редких частотах соударений
}

\author{
() В.К. Пашнев, Э.Л. Сороковой, А.А. Петрушеня, Ф.И. Ожерельев \\ Национальный научный центр „Харьковский физико-технический институт“, Институт фризики плазмы, \\ 61108 Харьков, Украина \\ e-mail: sorokovoy@ipp.kharkov.ua
}

Поступило в Редакцию 5 марта 2018 г.

В окончательной редакции 2 октября 2018 г.

Принято к публикации 21 ноября 2018 г.

В торсатроне Ураган-3М (У-3М) были проведены исследования флуктуаций полоидальной компоненты магнитного поля плазмы в диапазоне частот $0.5-50 \mathrm{kHz}$. Водородная плазма создается и нагревается ВЧполями на частотах, близких к ионной циклотронной. Исследования были выполнены с помощью набора из 15 магнитных датчиков, установленных в одном из сечений тора. ВЧ-нагрев обеспечивал получение плазмы с редкими частотами столкновений и наличием бутстреп тока. Для исследований был выбран момент времени, когда наблюдается максимальная амплитуда магнитных флуктуаций и заметно выражена их связь с энергосодержанием плазмы. Наблюдается два типа колебаний. Первый тип - токовая структура вращается с определенной частотой в основном в направлении вращения электронов в магнитном поле, а амплитуда медленно меняется со временем (вращающаяся структура). Второй тип - пространственная структура не вращается, но ее амплитуда изменяется с определенной частотой (стоячая структура). Для структур с данным полоидальным волновым числом частота флуктуаций и вращений близки. Стоячие структуры колебаний с различными полоидальными волновыми числами в данном частотном диапазоне скоррелированы между собой. Максимальная амплитуда вращающихся структур наблюдается с $m=2$, а для стоячих структур - с $m=3$ и достигает значений $\tilde{B} \leq 0.3 \mathrm{G}$ в области удержания. Частота колебаний не зависит от полоидального волнового числа $m$ для исследованных случаев $m=0,1,2,3$.

DOI: 10.21883/JTF.2019.05.47464.104-18

\section{Введение}

Исследования флуктуаций магнитного поля, выполненные на различных тороидальных ловушках плазмы, стимулировали теоретические исследования. В настоящее время предсказано большое количество неустойчивостей, которые могут вызывать флуктуации магнитного поля. Это альфвеновские, дрейфово-альфвеновские и дрейфово-звуковые [1]. Кроме того, возможно возбуждение геодезических акустических мод GAM [2], тороидально индуцированных альфвеновских ТАE [3], различного типа бета-индуцированных альфвеновских ВАE [4] и дрейфово-акустических BTG-мод [5], параметрических неустойчивостей, связанных с нагревом плазмы [6] и т.д. Также методы нагрева влияют на возбуждение дрейфовых волн [7], которые при конечных значениях $\beta$ (отношение энергии плазмы к энергии удерживающего магнитного поля) могут сопровождаться флуктуациями магнитного поля. Наличие продольного тока в стеллараторной магнитной конфигурации может сопровождаться возбуждением токовых неустойчивостей (kink- и tearing-мод) [8].

Из перечисленных выше работ видно, что плазменные неустойчивости в тороидальных магнитных ловушках зависят от многих факторов и их поведение весьма разнообразно. Поэтому для понимания природы неустойчивостей необходимо получение максимально возможной экспериментальной информации о частотном диапазоне, пространственной структуре, а также параметрах плазмы и методов ее нагрева.

Для исследования структуры плазменных неустойчивостей удобно использовать набор магнитных датчиков, которые располагаются вне объема удержания плазмы. Набор магнитных датчиков позволяет получить информацию о частотах и пространственной структуре флуктуаций магнитного поля, связанных с этими неустойчивостями. Важнейшим недостатком этой магнитной диагностики является принципиальное отсутствие возможности определить область локализации неустойчивости в плазменном объеме. Поэтому для выяснения области локализации неустойчивости необходимо использовать другие диагностики.

Целью настоящей работы является получение информации с помощью набора магнитных датчиков о неустойчивостях в плазме торсатрона У-3М, создаваемой с помощью ВЧ-нагрева в условиях редких столкновений между частицами плазмы. Для исследования выбран момент времени, когда амплитуда флуктуаций магнитного поля достигает максимальных значений, а скорость роста энергосодержания - минимальна.

\section{Экспериментальные условия}

Исследования проводились в торсатроне У-3М [9] при величине магнитного поля на геометрической оси 


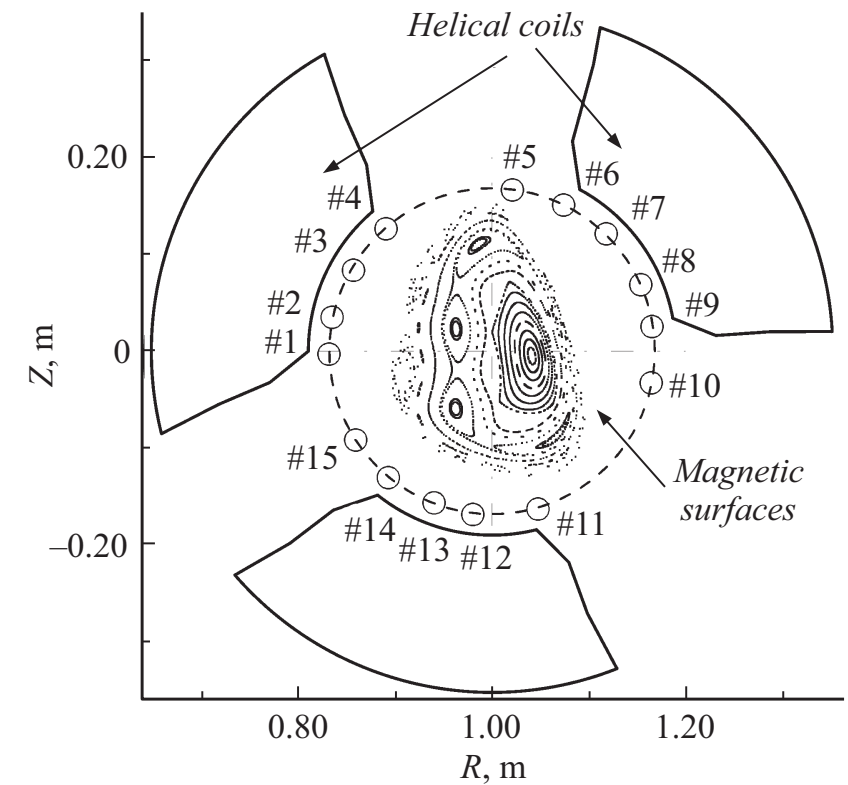

Рис. 1. Расположение магнитных датчиков относительно винтовых проводников магнитного поля и магнитных поверхностей вакуумной конфигурации в данном сечении.

плазменной конфигурации $B_{0} \approx 0.72$ Т. Малый усредненный радиус плазмы $a \approx 0.1 \mathrm{~m}$, а большой радиус тора $R \approx 1 \mathrm{~m}$. Плазма создавалась и нагревалась в режиме ВЧ-нагрева [10] на частотах, близких к ионной циклотронной частоте $\omega \approx 0.8 \omega_{c i}$, где $\omega_{c i}-$ циклотронная частота ионов рабочего газа. В качестве рабочего газа использовался водород. Согласно теории, основным механизмом нагрева плазмы является черенковское затухание волн, возбуждаемых в плазме в условиях альфвеновского резонанса, на электронах [11]. Очевидно, что такой способ передачи энергии электронам плазмы способствует, особенно при редких частотах столкновений, искажению функции распределения и появлению дополнительных условий возбуждения неустойчивостей. Так как возбуждаемые в плазме ВЧ-волны имеют частоту, близкую к ионной циклотронной, возможен дополнительный нагрев ионов с соответствующими искажениями функции распределения.

Для нас основной интерес представляет режим с низкой средней плотностью плазмы $n_{e} \leq 1 \cdot 10^{18} \mathrm{~m}^{-3}$, c максимальной плотностью на магнитной оси $n_{e}(0) \approx 3 \cdot 10^{18} \mathrm{~m}^{-3}[12,13]$. В этом режиме в момент исследования структуры флуктуаций магнитного поля средние по сечению плазменного шнура температура электронов $T_{e} \approx 150 \mathrm{eV}$ и ионов $T_{i} \approx 100 \mathrm{eV}$ [14], а ток в плазме составлял $I \approx 1 \mathrm{kA}$. Угол вращательного преобразования, создаваемый этим током на границе плазменного шнура, был в этот момент $l(a) \approx 3 \cdot 10^{-2}$, что существенно меньше стеллараторного угла вращательного преобразования $l_{s t} \approx 0.33$. Этот режим интересен тем, что электроны и ионы плазмы по частотам столкновений находятся в „банановом“ режиме, что подтверждается регистрацией так называемого бутстреп тока [15].

Информация о плазменных неустойчивостях была получена с помощью набора из 15 магнитных датчиков (катушек), регистрирующих полоидальную компоненту магнитного поля и установленных в одном из сечений плазменного шнура (рис. 1). Датчики имели диаметр $6 \mathrm{~mm}$ и длину $16 \mathrm{~mm}$, чувствительность $N_{S}=180 \mathrm{~cm}^{2}$ turns $(N-$ число витков в катушке, $S$ - площадь сечения катушки) и были расположены внутри электростатического экрана. Каждая катушка с подводящим кабелем длинной около $20 \mathrm{~m}$ позволяла регистрировать изменение магнитного поля с частотой до $70 \mathrm{kHz}$. Сигналы с датчиков интегрировались с помощью 16-канального электронного интегратора. Постоянная интегрирования варьировала в диапазоне $\tau=5 \cdot 10^{-7}-10^{-3} \mathrm{~s}$.

Кроме магнитных датчиков для контроля параметров разряда использовались пояс Роговского и диамагнитная петля.

Для исследований структуры магнитных флуктуаций (МФ) выбирался момент максимальной амплитуды колебаний, который характеризуется замедлением роста энергосодержания плазмы и появлением особенностей на токе. Резкое уменьшение амплитуды МФ сопровождается возрастанием скорости роста параметров плазмы, который в работах $[16,17]$ называется переходом в режим улучшенного удержания.

Энергия, запасенная в плазме к концу разряда, составляет около $16 \mathrm{~J}$, а энергия, запасенная в магнитном поле плазменного тока, - $6 \mathrm{~J}$, т.е. около $40 \%$ от энергии плазмы. Это указывает на возможность возникновения существенных неустойчивостей, связанных с наличием тока [8].

\section{Экспериментальные результаты}

На рис. 2 приведено временное поведение энергосодержания плазменного шнура $P$, тороидального плазменного тока $I$ и сигнала с одного из 15 магнитных датчиков, установленных вне области удержания на измерительной поверхности радиусом $b=16.8 \mathrm{~cm}$. Из рис. 2 видно, что к $15 \mathrm{~ms}$ от начала разряда происходит заметное увеличение амплитуды колебаний магнитного поля, а затем ее резкое уменьшение в несколько раз. При росте флуктуаций магнитного поля скорость роста энергосодержания падает. Уменьшение амплитуды колебаний совпадает с резким увеличением скорости роста энергосодержания плазмы. Этот момент на рис. 2 отмечен штриховой линией.

Временное поведение сигнала одного из датчиков в интервале 13.5-15.5 ms приведено на рис. 3. Из рисунка видно, что флуктуации магнитного поля в данном временном интервале представляют собой набор флуктуаций различной частоты с меняющейся во времени амплитудой и фазами. А именно регистрируемые сигналы 


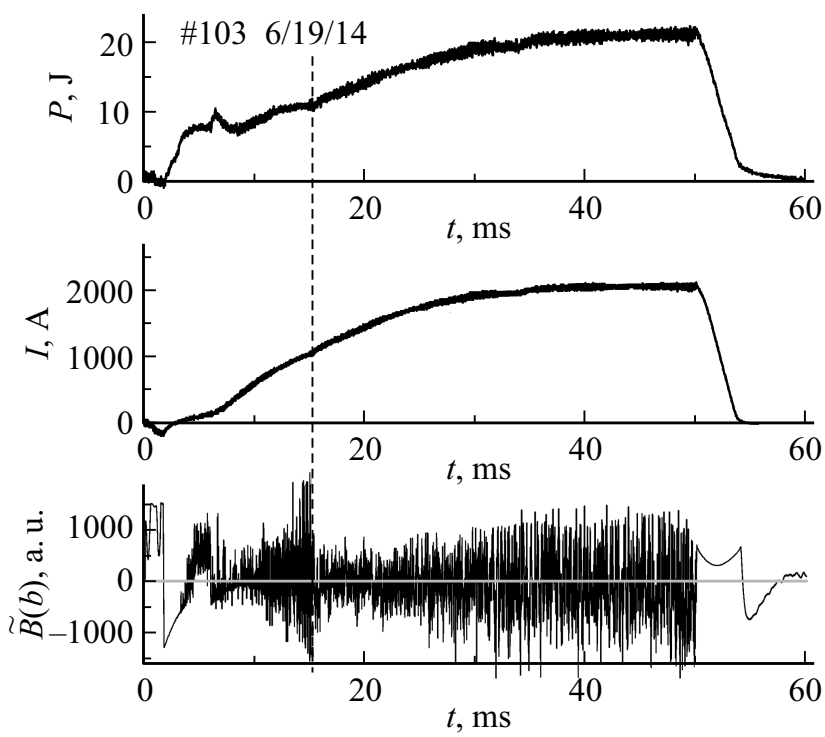

Рис. 2. Временное поведение энергосодержания в плазменном шнуре $P$, тороидального плазменного тока $I$ и сигнала, регистрируемого одной из катушек $\tilde{B}(b)$. Штриховой линией обозначен момент резкого уменьшения амплитуды колебаний магнитного поля $(t=15.3 \mathrm{~ms})$.
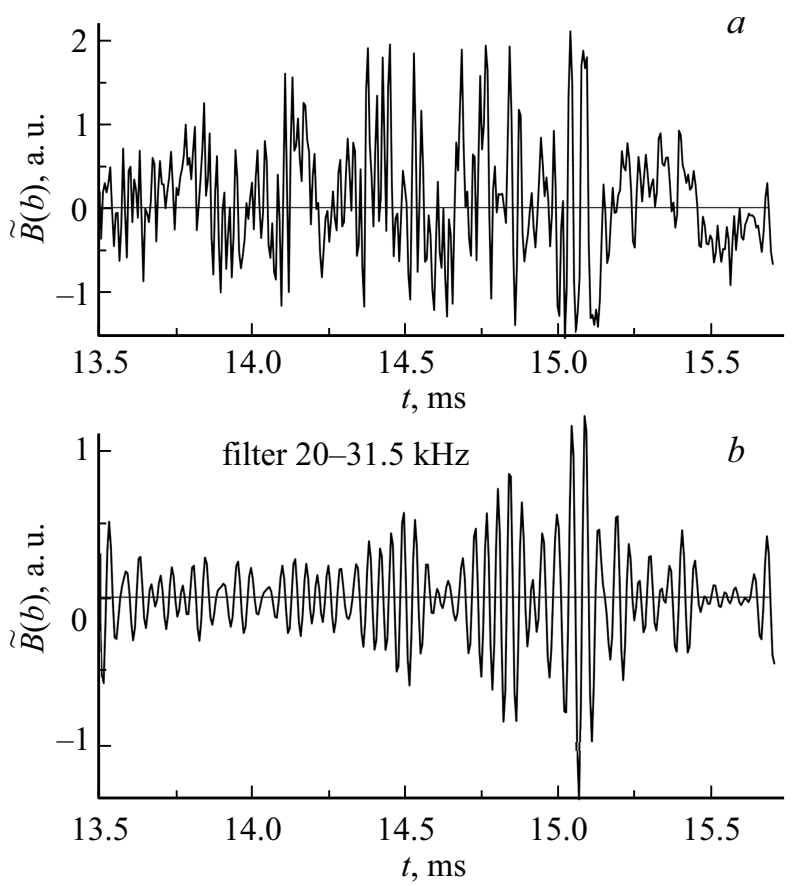

Рис. 3. Временное поведение флуктуаций магнитного поля, зарегистрированных одним из датчиков в определенном временном интервале: $a$ - реальная реализация; $b-$ эта же реализация после полосового фильтра в диапазоне $20-31.5 \mathrm{kHz}$.

имеют явно не стационарный характер, и использование стандартных методик для его обработки невозможно.

В связи с тем, что говорить в данном случае о локальной частоте некорректно, будем оперировать понятием полоса частот.
Для обработки полученного набора сигналов реализации были разбиты на 5 частотных диапазонов: $\delta f_{1}=0.5-5 \mathrm{kHz}, \quad \delta f_{2}=5-11 \mathrm{kHz}, \quad \delta f_{3}=11-20 \mathrm{kHz}$, $\delta f_{4}=20-31.5 \mathrm{kHz}, \delta f_{5}=31.5-52 \mathrm{kHz}$. Разделение на эти полосы частот проводилось по спектрам реализаций всех датчиков. Оцифрованные сигналы с помощью полосовых фильтров представлялись в виде реализации в определенном частотном диапазоне. На рис. 3 в качестве примера приведена реализация в частотном диапазоне $\delta f_{4}=20-31.5 \mathrm{kHz}$. Из рисунка видно, что данная реализация представляет собой набор колебаний с приблизительно одинаковым периодом. Амплитуда этих колебаний изменяется от минимальных до максимальных значений в течение двух-трех колебаний. А сам процесс изменения амплитуды колебаний состоит из последовательных циклов роста и уменьшения амплитуды за 5-7 колебаний, причем в каждом следующем цикле амплитуда колебаний становится больше. Как видно из рис. 3, таких циклов наблюдается 3, после чего с момента времени $15.3 \mathrm{~ms}$ циклы не повторяются. В других частотных полосах количество циклов может изменяться от 1 до 5. Исследовать структуру флуктуаций будем в последнем цикле перед падением уровня МФ.

Дальнейшая процедура обработки сигналов была следующей. В каждый момент времени в данном частотном диапазоне строилось азимутальное распределение величины сигнала по всем датчикам. Пример такого распределения для двух соседних моментов времени в полосе частот $20-31.5 \mathrm{kHz}$ приведен на рис. 4. Из рисунка видно, что полоидальное распределение величины колебаний магнитного поля на измерительной поверхности в данный момент времени имеет сложный вид. Кроме того, во времени это распределение изменяется. Наблюдается вращение структуры.

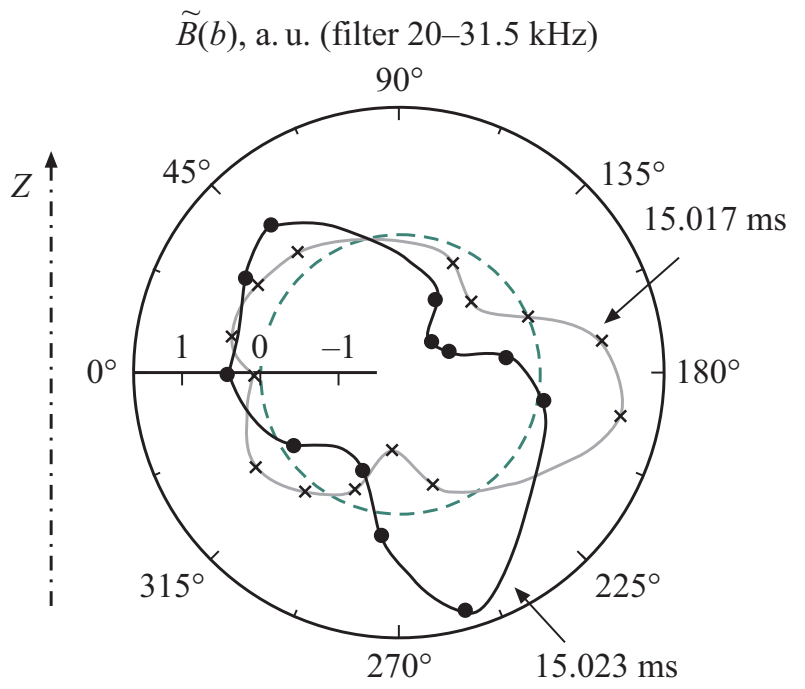

Рис. 4. Распределение величины сигналов с магнитных датчиков по азимуту для двух времен $t=15.017$ и $15.023 \mathrm{~ms}$ для реализации в полосе частот $20-31.5 \mathrm{kHz}$. Штрихом на данном рисунке показан нулевой уровень, штрихпунктир — ось тора $Z$. 


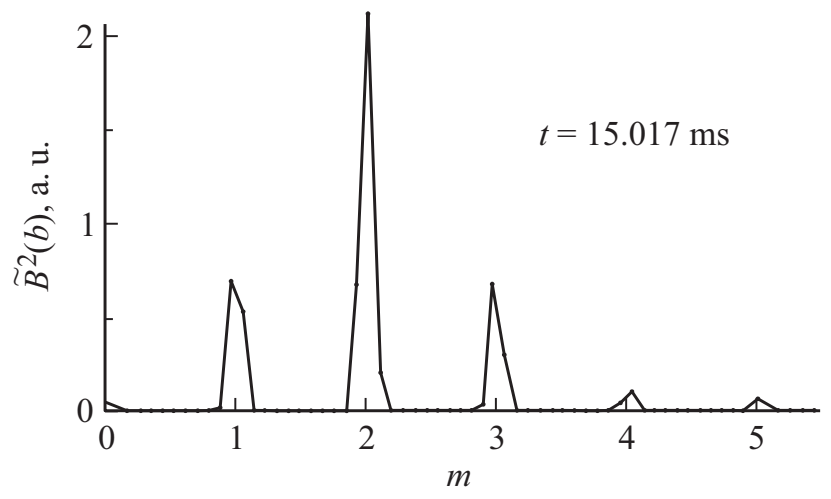

Рис. 5. Спектр разложения по полоидальным гармоникам $\mathrm{m}$ флуктуаций магнитного поля $(t=15.017 \mathrm{~ms}$, $\left.\delta f_{4}=20-31.5 \mathrm{kHz}\right)$.

В дальнейшем было проведено разложение пространственной структуры распределения величины сигнала на фурье-гармоники. Пример такого разложения приведен на рис. 5 для момента времени $t=15.017 \mathrm{~ms}$ в полосе частот 20-31.5 kHz. Полученная картина представляется в виде набора гармоник с различными полоидальными волновыми числами $m=0,1,2,3$ и т.д. Более высокие гармоники с волновыми числами $m>3$ в дальнейшем не учитывались, так как точность их определения с помощью неравномерно расположенных 15 датчиков невелика, а также невелика их амплитуда. Кроме амплитуды данной пространственной гармоники регистрировалась также ее фаза относительно предыдущего момента времени.

С физической точки зрения гармоника с $m=0$ представляет собой колебания магнитного поля продольного плазменного тока; $m=1$ - это колебания дипольного тока, аналогичного току Пфирша-Шлютера, повернутого в пространстве на любой произвольный угол. Структура с $m=2$ - это эллипс, а с $m=3$ - треугольник.

Следует обратить внимание на тот факт, что рассмотренное представление полностью справедливо лишь в цилиндрической геометрии. В реальном, хоть и пологом торе, а также при наличии смещения токовой поверхности относительно измерительной и некруглой формы токовой поверхности, необходимо учитывать взаимную связь соседних полоидальных гармоник.

В цилиндрической геометрии знание величины амплитуды определенной полоидальной гармоники магнитного поля на измерительной поверхности позволяет определить величину магнитного поля на токовой поверхности. Известно, что в этом случае спад величины магнитного поля данной гармоники описывается соответствующей функцией Макдональда [18]. В первом приближении спад амплитуды различных гармоник пропорционален величине $\left(r_{0} / b\right)^{m+1}$. Здесь $r_{0}-$ средний радиус токовой поверхности, а $b-$ радиус измерительной поверхности.
Уравнение магнитной поверхности можно записать в цилиндрической системе координат $r, \vartheta$ :

$$
r_{0}=r+\Delta \cos \vartheta-\delta \cos 2 \vartheta
$$

Здесь $r$ - текущий радиус, $\vartheta-$ полоидальный угол, a $\Delta$ - смещение и $\delta$ - эллиптичность магнитной поверхности, на которой протекают токи. Как видно из рис. 1, треугольность магнитных поверхностей мала, и ее можно не учитывать. Выражение для величины магнитного поля на измерительной поверхности с учетом тороидальности [18] можно представить в виде

$$
\begin{aligned}
B_{m}(b) & \cos (m \vartheta+\psi)=\sum_{n=0}^{3} B_{n}\left(r_{0}\right)\left(\frac{r}{b}\right)^{n+1} \\
\times & {\left[1+\frac{\Delta}{r} \cos \vartheta+\frac{\delta}{r} \cos 2 \vartheta\right]^{n+1} } \\
& \times \sqrt{1-\frac{r}{R} \cos \vartheta} \cos (n \vartheta+\psi),
\end{aligned}
$$

где $\psi-$ произвольная фаза.

Если величины $\frac{\Delta}{r} ; \frac{\delta}{r} ; \frac{r}{R} \ll 1$ и, предполагая, что амплитуда 3-й гармоники намного превышает амплитуду 4-й гармоники, что не противоречит данным эксперимента, то выражение (2) можно представить в виде системы из 4-х линейных уравнений для $m=0,1,2,3$.

Предполагаем, что источник колебаний находится внутри объема удержания на магнитной поверхности со средним радиусом $r_{0}=8.4 \mathrm{~cm}$. Для этой поверхности определяются величины $\Delta$ и $\delta$, и на основании имеющихся экспериментальных данных был проведен расчет амплитуды флуктуаций для каждой гармошки на магнитной поверхности, по которой протекают токи. Проведенные расчеты показывают, что в наших условиях учет смещения и эллиптичности магнитной поверхности, а также тороидальности, сильно не влияют на величину амплитуды поля внутри объема удержания.

Результаты обработки зарегистрированных сигналов приведены на рис. 6. В качестве примера выбран частотный диапазон $\delta f_{3}=11-20 \mathrm{kHz}$. На рисунке для каждой пространственной гармоники представлены изменение во времени фазы $\vartheta$ в диапазоне $-\pi-\pi$ и ее амплитуда. На оси $Y$ отложена величина $\tilde{B}^{2}$.

Как видно из приведенных на рис. 6 данных, изменение фазы структуры с $m=0$ представляют собой величины 0 и $\pi$, что соответствует противоположным фазам колебаний. По величине периода колебаний можно оценить полосу частот, в которой происходят колебания. Так, в период времени $t=14.9-15.0 \mathrm{~ms}$ по периоду колебаний можно оценить частоту $f \approx 14 \mathrm{kHz}$; близкую частоту имеют колебания в период времени $t=15.05-15.13 \mathrm{~ms}$. Для структуры с $m=1$ картина более сложная. Если рассматривать с момента времени $t=14.9 \mathrm{~ms}$, то сначала наблюдается стоячая на месте структура, амплитуда которой меняется с частотой $f \approx 14 \mathrm{kHz}$. Однако при приближении величины 

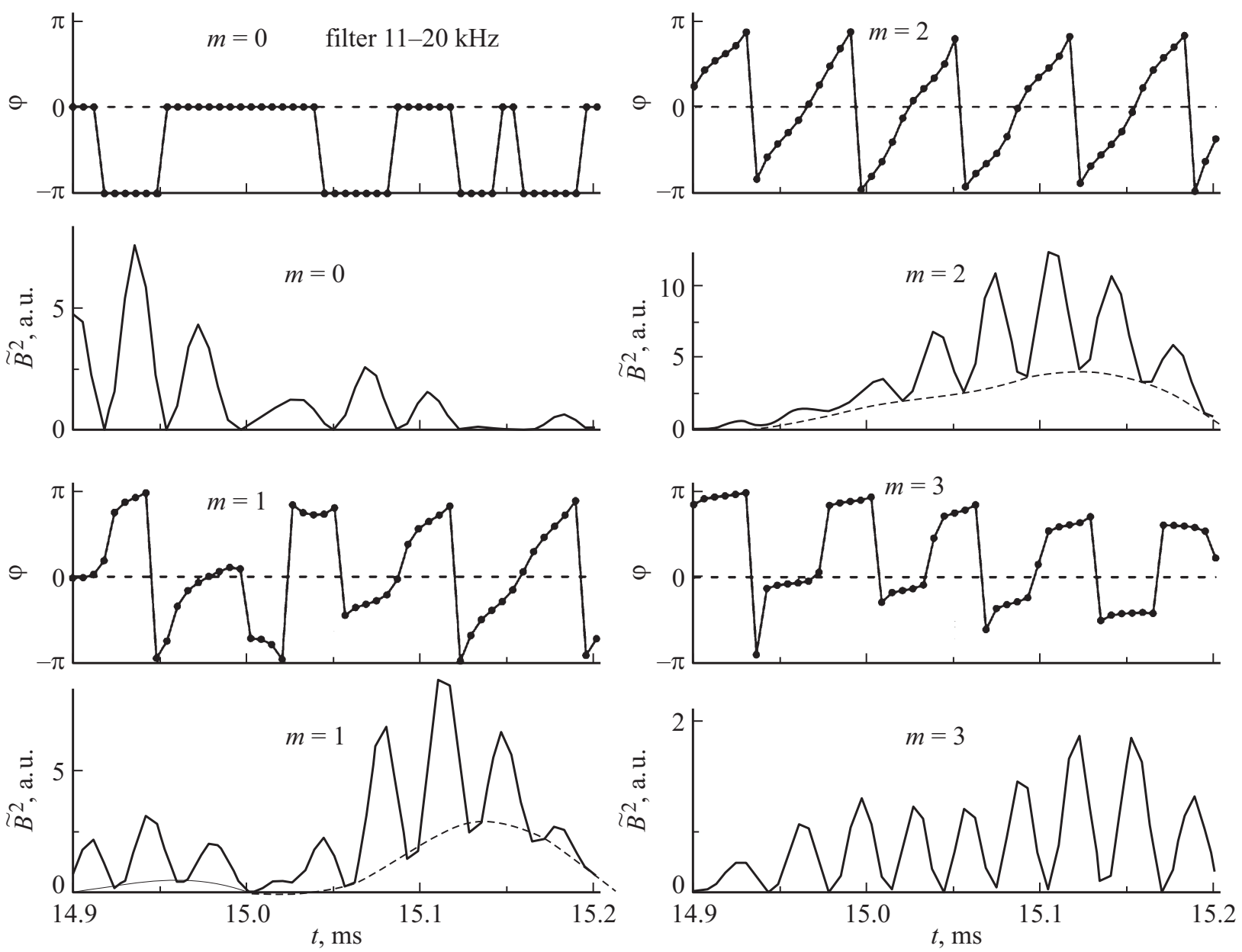

Рис. 6. Зависимость амплитуды и фазы структур с полоидальными волновыми числами $m=0,1,2,3$ для частотного диапазона $\delta f_{3}=11-20 \mathrm{kHz}$. Штрихом показана амплитуда вращающейся структуры.

колебаний к нулю, судя по сдвигу фаз, структура начинает медленно поворачиваться в сторону вращения электронов в магнитном поле. Через период колебаний структура начинает вращаться в противоположном направлении, а потом и вовсе останавливается. В дальнейшем $t=15.05-15.2 \mathrm{~ms}$, снова появляется структура, в которой наблюдаются колебания с периодом, соответствующим частоте $f \approx 14 \mathrm{kHz}$. В это же время появляются вращающиеся колебания с медленно меняющейся амплитудой. Ее величина может быть определена по величине огибающей минимальных значений колебаний и обозначена штриховой линией. Видно, что наблюдаются 2 структуры плазменных токов с $m=1$. Одна структура вращается с частотой $f \approx 13.5 \mathrm{kHz}$ в сторону электронного дрейфа, ее амплитуда медленно изменяется во времени (штриховая линия). И другая структура, которая стоит на месте (не вращается), ее амплитуда колеблется с частотой $f \approx 14 \mathrm{kHz}$. Для колебаний магнитного поля с полоидальным волновым числом $m=2$ ситуация аналогичная. Амплитуда стоячей структуры колеблется с частотой $f \approx 14 \mathrm{kHz}$, вращающаяся - вращается с переменной угловой скоростью, соответствую- щей диапазону частот $f \approx 14-18 \mathrm{kHz}$. Для структуры с $m=3$ в данном частотном диапазоне $\delta f_{3}=11-20 \mathrm{kHz}$ наблюдаются только колебания, которые стоят на месте (не вращаются). Частота колебаний около $f \approx 14.5 \mathrm{kHz}$.

Анализ всех имеющихся экспериментальных данных показывает, что в данном эксперименте в частотном диапазоне $f \approx 0.5-52 \mathrm{kHz}$ наблюдаются 2 типа колебаний. Первый тип - структура с данным волновым числом стоит на месте (не вращается) или очень медленно вращается. С регистрируемой частотой изменяется только ее величина. Такого типа колебания наблюдаются всегда. Второй - структура вращается с определенной частотой, а ее амплитуда слабо изменяется. Частота вращения чаще всего не постоянна во времени. Обычно, частота колебаний стоячей структуры близка к частоте вращения структуры второго типа. Если учесть, что стоячая структура при приближении ее величины к нулю может быстро повернуться на определенный угол, можно предположить, что стоячая структура стимулирует вращение.

Максимальная амплитуда флуктуаций в исследуемом процессе для пространственных структур с 
$m=0,1,2,3$ для всех регистрируемых частот приведена на рис. 7. Положительные и отрицательные значения амплитуды соответствуют разным направлениям вращения структуры (заштрихованные столбики). Положительное значение соответствует вращению в направлении вращения электронов в магнитном поле. Не заштрихованные столбики определяют максимальную амплитуду стоячей структуры. Ширина столбика показывает приблизительный частотный диапазон регистрируемых флуктуаций.

Анализ приведенных экспериментальных данных показывает, что флуктуации магнитного поля в данном эксперименте существуют в достаточно узких частотных диапазонах, общих для пространственных структур с различными полоидальными волновыми числами. Флуктуации магнитного поля зарегистрированы в таких частотных диапазонах: $1.5-2 ; 6-8 ; 13-16 ; 20-27$; 35-43 kHz (рис. 7).

Для структур с $m=1$ и 2, в диапазоне частот $1.5-2 \mathrm{kHz}$ наблюдается вращение в различных направлениях, определить амплитуды которых не удалось. Максимальные величины флуктуаций магнитного поля стоячих структур наблюдаются для $m=2$ и 3 .

В диапазоне частот $6-8 \mathrm{kHz}$ наблюдаются флуктуации как стоячих структур с $m=0,1,2,3$, так и вращающихся структур с $m=1,2,3$. Следует отметить, что все флуктуации стоячих структур связаны между собой. А именно их частоты и фазы близки между

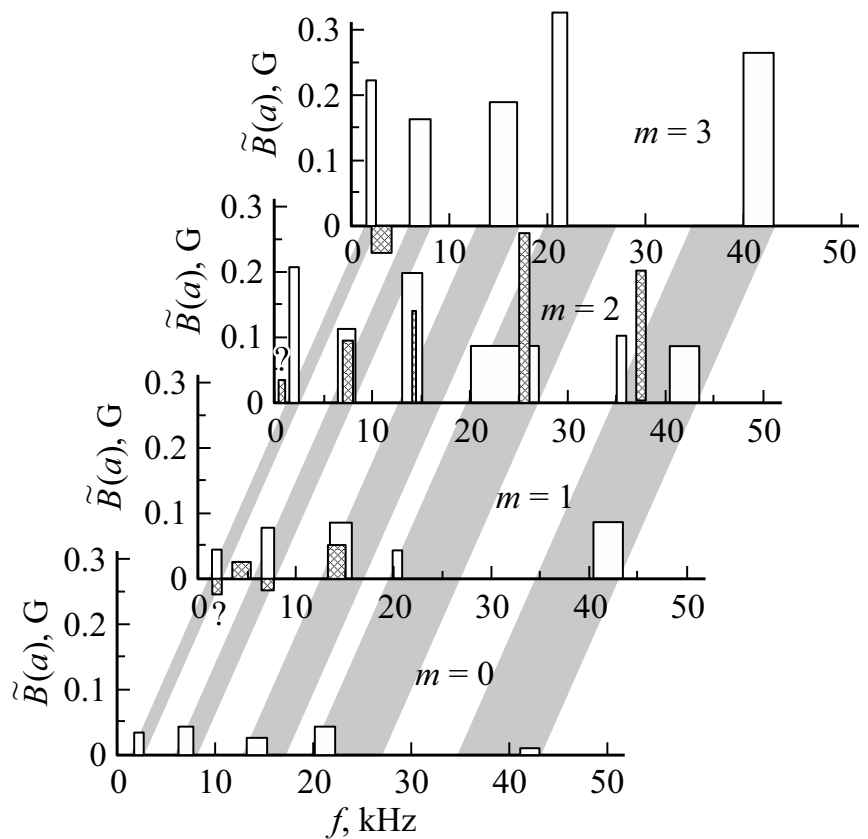

Рис. 7. Зависимость максимальной амплитуды флуктуаций от регистрируемой полосы частот для пространственных структур с различными полоидальными волновыми числами $m$. Светлые столбики определяют стоячие структуры, а заштрихованные - вращающиеся. Положительная величина заштрихованных столбиков соответствует направлению вращения электронов в магнитном поле, а отрицательная — ионов.

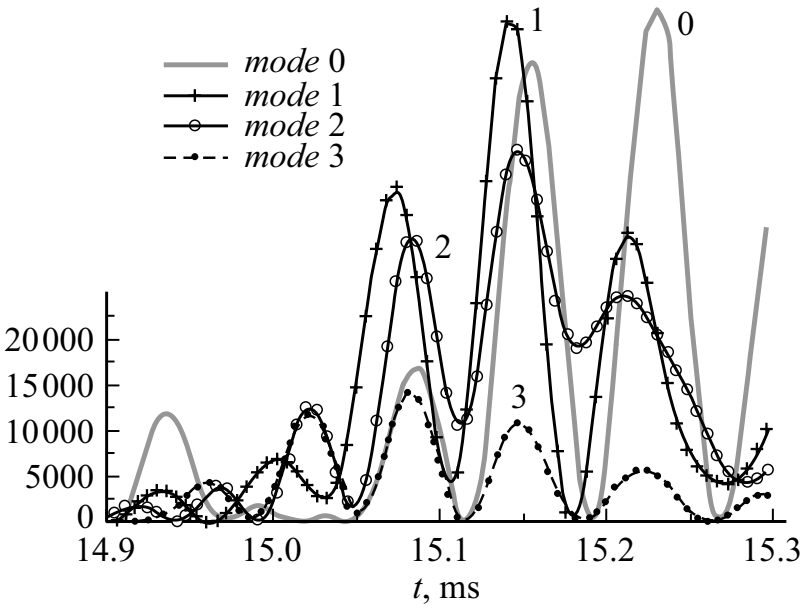

Рис. 8. Амплитуды флуктуаций для четырех пространственных структур с $m=0,1,2,3$ в частотном диапазоне $5-11 \mathrm{kHz}$ на измерительной поверхности.

собой и представляют собой общее возмущение для всех пространственных структур (рис. 8). Амплитуда колебаний внутри области удержания растет с номером полоидальной моды (рис. 7). Максимальную амплитуду имеют вращающиеся структуры с $m=2$.

Для флуктуаций, наблюдаемых в диапазоне $13-16 \mathrm{kHz}$, вращение присутствует только для структур c $m=1$ и 2. Стоячие структуры коррелируют между собой (кроме $m=0$ ) и имеют максимальную амплитуду для структур с $m=2$ и 3. Амплитуды вращающихся структур в этом диапазоне частот меньше амплитуд стоячих структур.

В исследуемой полосе $\delta f_{4}=20-31.5 \mathrm{kHz}$ флуктуации стоячих структур находятся в диапазоне $20-22 \mathrm{kHz}$, кроме структуры $m=2$, полоса которой составляет 20-27 kHz. Амплитуда структуры с $m=3$ достигает максимального значения $\tilde{B}\left(r_{0}\right) \approx 0.3 \mathrm{G}$. Вращается только структура $m=2$ с частотой $f_{\text {spin }}=25-26 \mathrm{kHz}$, причем амплитуда вращающейся структуры заметно превосходит амплитуду стоячей структуры.

В полосе $\delta f_{5}=31.5-52 \mathrm{kHz}$ флуктуации стоячих структур находятся в диапазоне $40-43 \mathrm{kHz}$, кроме структуры с $m=2$, где наблюдаются флуктуации в полосе $35-37 \mathrm{kHz}$ и частотой вращения $f_{\text {spin }} \approx 37 \mathrm{kHz}$. Для $m=2$ амплитуда вращающейся структуры выше стоячей структуры. В этом диапазоне частот вращается только структура с $m=2$. Максимальную амплитуду имеют колебания стоячей структуры с $m=3$, которые внутри области удержания достигают величины $\tilde{B}\left(r_{0}\right) \approx 0.25 \mathrm{G}$.

\section{Обсуждение полученных результатов}

Как видно из рис. 7, в исследуемый момент времени регистрируются МФ в диапазоне частот $f=1.5-43 \mathrm{kHz}$. Причем эти флуктуации находятся в пяти узких частотных диапазонах, так, что $\Delta f / f \leq 0.25$. 


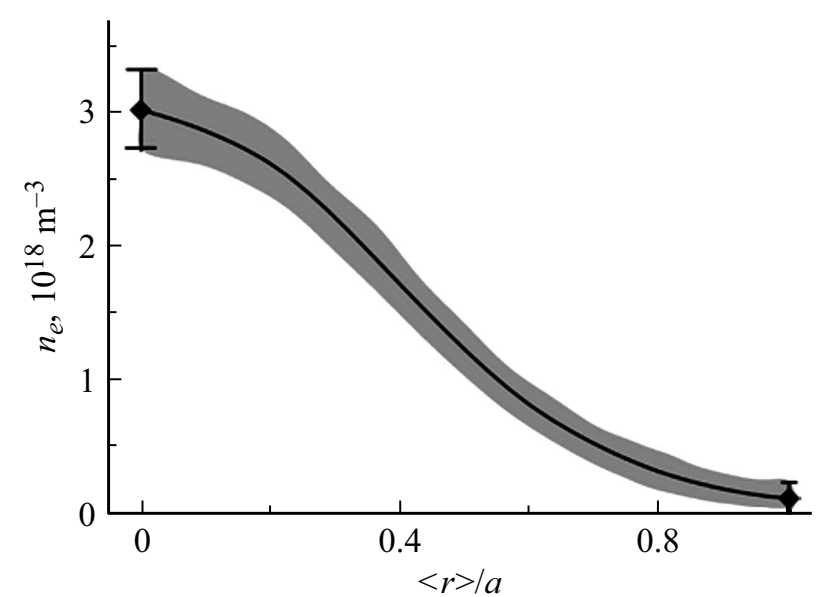

Рис. 9. Распределение плотности плазмы в момент исследования структуры магнитных флуктуаций, $\langle r\rangle-$ средний радиус магнитной поверхности.

В каждом из этих частотных диапазонов частота флуктуаций не зависит от полоидального волнового числа $m$. Наблюдаются 2 типа флуктуаций: стоячие структуры и вращающиеся в полоидальном направлении структуры с определенным $m$. Амплитуды колебаний стоячих структур растут с ростом $m$.

Следует отметить, что наблюдается хорошая корреляция между стоячими колебаниями с волновыми числами $m=1,2$ и 3 во всех частотных диапазонах. В диапазоне частот $f_{r}=6-8 \mathrm{kHz}$ наблюдается корреляция даже с модой $m=0$ (рис. 8).

Вращающиеся структуры наблюдаются для всех волновых чисел $m=1,2,3$ (кроме $m=0$ ). Только для структуры с $m=3$ амплитуда слишком мала. Вращение направлено в основном в сторону электронногодиамагнитного дрейфа, кроме случая низких частот для $m=3$. Наибольшая амплитуда вращающейся структуры наблюдается для $m=2$. В этом случае амплитуда вращающейся структуры может превосходить амплитуду стоячей структуры. Частота вращения обычно близка частоте колебаний стоячей структуры.

Рассмотрим частоты, характерные для плазмы в исследуемом диапазоне частот. В условиях редких частот столкновений запертые частицы описывают траекторию с характерной частотой:

$$
f_{j}^{*}=\frac{v_{T j} l}{2 \pi R}\left(\frac{r}{R}\right)^{3 / 2}
$$

где $v_{T j}$ - тепловая скорость частиц $j$-сорта, $l-$ угол вращательного преобразования, $r$ - текущий радиус. Для электронов, согласно (3), $f_{e}^{*} \approx 5 \cdot 10^{2} \mathrm{~Hz}$ и для ионов $f_{i}^{*} \approx 3.5 \cdot 10^{3} \mathrm{~Hz}$. Эти частоты находятся ниже или вблизи нижней границы регистрируемых в эксперименте частот.

\begin{tabular}{c|c|c|c|c}
\hline$r / a$ & 0.6 & 0.7 & 0.8 & 0.9 \\
\hline$f_{d r}, \mathrm{kHz}$ & 25 & 29 & 40 & 61
\end{tabular}

Выражение для частоты диамагнитного дрейфа можно представить в виде

$$
f_{d r}=-\frac{m}{2 \pi r} \frac{c \frac{\partial p}{\partial r}}{e n_{e} B} .
$$

Здесь $p=n_{e} k\left(T_{e}+T_{i}\right)$ - давление плазмы. Если считать, что распределение температуры по радиусу однородно, то для оценок можно воспользоваться распределением плотности, измеренным в аналогичном режиме [12] (рис. 9). График построен по двум измеренным значениям локальной плотности с помощью ленгмюровского зонда на границе области удержания и по прохождению СВЧ-волн на разных частотах в центре плазмы с учетом того, что интеграл по профилю должен быть равен средней плотности плазмы, измеренной интерферометром. Это распределение достаточно хорошо аппроксимируется выражением $n_{e}=n_{e}(0)\left[1-(r / a)^{1.5}\right]^{2}$. Тогда для $n_{e}(0) \approx 3 \cdot 10^{18} \mathrm{~m}^{-3}$, согласно выражению (4), для $m=1$ получим расчетные данные, представленные в таблице.

Видно, что для $m=1$ дрейфовые частоты находятся в измеряемом диапазоне частот. Однако для $m=2$ и 3 они существенно превосходят регистрируемые в эксперименте. Если учесть неоднородность распределения температуры, то значения еще больше возрастут.

Геодезическая акустическая мода (GAM) с учетом средней кривизны магнитного поля дает значение частоты [2]:

$$
f_{\mathrm{GAM}}=\frac{v_{s}}{2 \pi R} \sqrt{2+l^{2}(a)} \approx 46 \mathrm{kHz}
$$

где $v_{s}$ - звуковая скорость. Как видно из выражения (5), частота $f_{\text {GAM }}$ близка к верхней границе регистрируемых частот.

Частота ТАЕ-моды (Toroidal Induces Alfen Eigen Mode) [3] в наших условиях составляет

$$
f_{\mathrm{TAE}}=\frac{v_{A} l}{4 \pi R} \approx 50 \mathrm{kHz},
$$

где $v_{A}$ - альфвеновская скорость. То есть частота ТАЕмоды тоже находится на верхней границе регистрируемых частот.

В плазме возможно также возбуждение так называемых ВАЕ-мод (beta induced alfen modes) [7], которые являются следствием связи идеальных МГД альфвеновских дрейфовых и акустических мод на частотах,

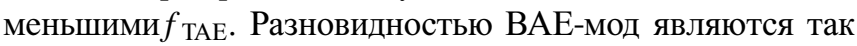
называемые BTG (beta induced temperature gradient) моды, которые были получены при учете теплового 
движения ионов [5]. Для этих мод характерны частоты

$$
f_{\mathrm{BTG}} \leq \frac{v_{T i} l}{2 \pi R} \approx 6 \mathrm{kHz} \text { и } f_{\mathrm{BTG}} \leq \frac{v_{t i} N}{2 \pi n l_{0}} \approx 57 \mathrm{kHz},
$$

которые являются тороидальными и трехмерными сателлитами. Здесь $l_{0}-$ заходность стеллараторного магнитного поля и $N$ - число периодов поля по длине установки. Условие $f_{\text {ВTG }}<f_{\text {TАЕ }}$ соблюдается только для случая $f_{\text {втG }} \leq \frac{v_{T i l} l}{2 \pi R} \approx 6 \mathrm{kHz}$. Такая неустойчивость возбуждается, если ионное $\beta_{i}=\frac{8 \pi n_{c} k T_{i}}{B^{2}}>\beta_{c r} \approx 4 \cdot 10^{-4}$ [5] для наших условий. Структура такой неустойчивости вращается в сторону ионного диамагнитного дрейфа. Условием развития данной неустойчивости является

$$
f_{d r}=f_{\mathrm{BTG}}=\frac{v_{t i} l}{2 \pi R} .
$$

В наших условиях $\frac{v_{t i l} l}{2 \pi R}=6 \mathrm{kHz}$, что не совпадает с данными для $f_{d r}$, приведенными в таблице. Частоты (7) совпадают со спектром измерений только на границах диапазона регистрируемых частот. Направление вращения структуры в противоположную сторону с данными эксперимента.

Наличие продольного тока в нашем эксперименте может быть причиной возбуждения токовых неустойчивостей (kink, tearing modes) [8], которые развиваются на рациональных магнитных поверхностях. В наших условиях это могут быть поверхности $l=0.25(\mathrm{~m} / n=4 / 1$; $8 / 2$ и т.д.), $l=0.33$ ( $r=10$ с $m / n=3 / 1 ; 6 / 2$ и т.д.) и промежуточные поверхности с $m>4$. Здесь $n-$ волновое число вдоль тора. В эксперименте возмущения c $m=3$ надежно регистрируются. Однако связать ее с развитием токовой неустойчивости очень трудно изза того, что плотность тока на границе $(r=10)$ равна нулю. Флуктуации с $m \geq 4$ нами регистрируются, но для 15 не равномерно расположенных датчиков трудно быть уверенным в достоверности такой информации, тем более что амплитуда таких возмущений мала.

Анализ, выполненный выше, показывает, что полученная информация о флуктуациях в плазме торсатрона У3М с трудом может быть объяснена существующей теорией. Это, прежде всего, наличие в полосе $1.5-50 \mathrm{kHz}$ пяти узкополосных частотных диапазонов и наличие одновременно стоячих и вращающихся структур с близкими частотами. Ближе всего к результатам измерений подходят так называемые GAM- и TAE-моды, частоты которых не зависят от полоидального волнового числа. Однако мы не располагаем теоретической информацией о направлении вращения этих мод и одновременного наличия среди них стоячих и вращающихся структур с близкими частотами. Кроме того, частотный диапазон этих мод не совпадает с данными эксперимента.

В работе [19] было замечено, что так называемый переход в состояние улучшенного удержания привязан к определенной величине тока. Так как наблюдаемый в наших экспериментах ток является бутстреп током, то наблюдается привязка к величине $\beta$. И всплеск амплитуды колебаний магнитного поля в исследуемом частотном диапазоне может наступать при превышении некоего предела по $\beta$. То есть наблюдаемые неустойчивости могут быть отнесены к так называемым - beta induced модам.

\section{Выводы}

Проведенные исследования показали, что в режиме редких столкновений между частицами плазмы при наличии бутстреп тока в условиях ВЧ-нагрева в торсатроне У-3М, в области частот до $50 \mathrm{kHz}$, в плазменном объеме возникают достаточно узкополосные МФ в пяти частотных диапазонах. Это диапазоны: $1.5-2$; 6-8; $13-16 ; 20-27 ; 35-43 \mathrm{kHz}$.

Пространственную структуру этих флуктуаций можно представить в виде полоидальных волновых чисел с $m=0,1,2,3$.

Частота наблюдений МФ не зависит от полоидального волнового числа.

Наблюдается два типа флуктуаций:

- стоячие на месте или очень медленно вращающиеся структуры, в которых магнитное поле меняется в определенной полосе частот;

- вращающиеся структуры, в которых амплитуда слабо изменяется, а максимальная частота вращения близка к частоте флуктуаций стоячей структуры с аналогичным полоидальным волновым числом.

Стоячие структуры МФ представляют собой сложную пространственную конфигурацию (флуктуации с различными волновыми числами коррелируют между собой) в данном частотном диапазоне. Эти структуры имеют максимальную амплитуду колебаний для волновых чисел с $m=3$ и достигают величины $\tilde{B} \approx 0.3 \mathrm{G}$ в области удержания.

Вращающиеся структуры могут иметь различные полоидальные числа, однако максимальные амплитуды имеют структуру с $m=2$. Направление вращения совпадает с вращением электронов в магнитном поле, за исключением диапазона частот $1.5-2 \mathrm{kHz}$, где вращения могут быть в обоих направлениях.

Невозможно полностью объяснить поведение флуктуаций магнитного поля в объеме удержания в рамках рассмотренных теоретических моделей.

Авторы выражают благодарность коллегам за полезное обсуждение и экипажу установки У-3М за обеспечение проведения эксперимента. Особую благодарность выражаем В.С. Войцене за полезные обсуждения и замечания в ходе работы над этой статьей.

\section{Список литературы}

[1] Михайловский А.Б. Неустойчивости плазмы в магнитных ловушках. М.: Атомиздат, 1978. 296 с.

[2] Winsor N., Johnson J.L., Dawson J.M. // Phys. Fluids. 1968. Vol. 11. N 11. P. 2448. 
[3] Cheng C.Z., Chen L., Chance M.S. // Ann. Phys. 1985. Vol. 161. P. 21

[4] Heidbrink W.W., Strait E.J., Chu M.S., Turnbull A.D. // Phys. Rev. Lett. 1993. Vol. 71. P. 855.

[5] Mikhailovskii A.B., Sharapov S.E. // Plasma Phys. Rep. 1999. Vol. 25. N 10. P. 803-816.

[6] Stepanov K.N. // Plasma Phys. Contr. F. 1996. Vol. 38. N 12A. P. A13.

[7] Heidbrink W.W. // Phys. Plasmas. 2008. Vol. 15. N 5. P. 055501.

[8] Sinclair R.M., Yoshikawa S., Harries W.L. et al. // Phys. Fluids. 1965. Vol. 8. P. 118.

[9] Chechkin V.V., Fomin I.P., Grigor'eva L.I. et al. // Nucl. Fusion. 1996. Vol. 36. N 2. P. 133.

[10] Chechkin V.V., Grigor'eva L.I., Pavlichenko R.O. et al. // Plasma Phys. Rep. 2014. Vol. 40. N 8. P. 601.

[11] Longinov A.V., Stepanov K.N. High-Frequency Plasma Heating / Ed. by A.G. Litvak. NY: American Institute of Physics, 1992. P. 93-238.

[12] Pashnev V.K., Tarasov I.K., Sitnikov D.A. et al. // Probl. Atom. Sci. Tech. 2013. Vol. 1. P. 15.

[13] Kasilov A.A., Grigor'eva L.I., Chechkin V.V. et al. // Probl. Atom. Sci. Tech. 2015. Vol. 1. P. 24.

[14] Pashnev V.K., Sorokovoy E.L., Petrushenya A.A. et al. // Probl. Atom. Sci. Tech. 2010. Vol. 6. P. 24.

[15] Pashnev V.K., Sorokovoy E.L. // Probl. Atom. Sci. Tech. 2008. Vol. 6. P. 31-33.

[16] Chechkin V.V., Grigor'eva L.I., Sorokovoy Ye.L. et al. // Plasma Phys. Rep. 2009. Vol. 35. N 10. P. 852.

[17] Pashnev V.K., Sorokovoy E.L., Berezhnyj V.L. et al. // Probl. Atom. Sci. Tech. 2010. Vol. 6. P. 17-20.

[18] Морозов А.И., Соловьев Л.С. Вопросы теории плазмы. Вып. 2. М.: Атомиздат, 1963. С. 51, 70.

[19] Dreval M.B., Yakovenko Yu.V., Sorokovoy E.L. et al. // Phys. Plasmas. 2016. Vol. 23. P. 022506. 